\title{
Thermal smoothing and roughening of GaAs surfaces: experiment and Monte Carlo simulation
}

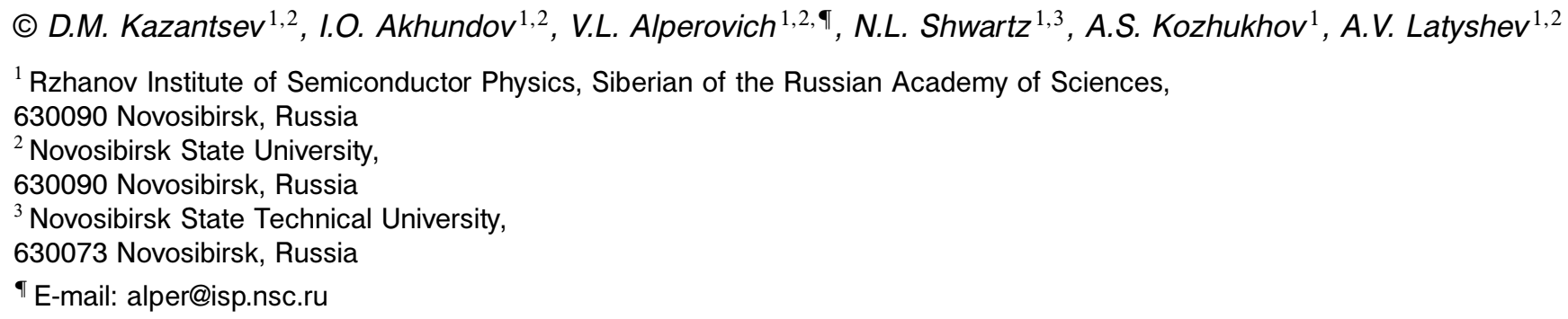

GaAs thermal smoothing at temperatures $T \leq 650^{\circ} \mathrm{C}$ in the conditions close to equilibrium yields surfaces with atomically smooth terraces separated by steps of monatomic height. At higher temperatures surface smoothing is changed to roughening. Possible reasons of surface roughening at elevated temperatures are studied by means of Monte Carlo simulation and compared with the experimental results on GaAs. It is proved that GaAs roughening at elevated temperatures is caused by kinetic instabilities due to deviations from equilibrium towards growth or sublimation. The microscopic mechanisms of kinetic-driven roughening are discussed.

\section{Acknowledgements}

The AFM measurements and Monte-Carlo simulations were supported by RSF (Grant No 14-22-00143). 